\title{
Frequencies of gamma/beta oscillations are stably tuned to stimulus properties

\author{
Weijia Feng ${ }^{a, b}$, Martha N. Havenith ${ }^{c}$, Peng Wang ${ }^{a, b}$, Wolf Singer ${ }^{a, b}$ \\ and Danko Nikolić ${ }^{\mathrm{a}, \mathrm{b}}$
}

\begin{abstract}
Stimulus-induced changes in oscillation frequencies may affect information flow in the brain. We investigated whether the oscillation frequency of spiking activity in cat area 17 changes as a function of the drifting direction of sinusoidal gratings. Oscillation frequencies were tuned to specific drifting directions, such that some directions induced higher oscillation frequencies than others. When activity from the same neurons was recorded at a later time point, the average oscillation frequency with which the neurons responded had also often changed. However, the direction tuning of the neurons' oscillation frequencies remained constant. Thus, while the overall oscillation frequency, across all drift directions, was state-dependent, the relative change in oscillation frequencies induced by stimulus properties was not, the tuning remaining
\end{abstract}

stable. NeuroReport 21:680-684 (c) 2010 Wolters Kluwer Health | Lippincott Williams \& Wilkins.

NeuroReport 2010, 21:680-684

Keywords: area 17, beta/gamma oscillations, drifting gratings, oscillation frequency, visual cortex

${ }^{\mathrm{a}}$ Max Planck Institute for Brain Research, Deutschordenstraße, ${ }^{\mathrm{b}}$ Frankfurt Institute for Advanced Studies, Johann Wolfgang Goethe University, Frankfurt am Main, Germany and "Wolfson Institute for Biomedical Research, University College London, London, UK

Correspondence to Dr Danko Nikolić, PhD, Department of Neurophysiology, Max-Planck Institute for Brain Research, Deutschordenstr. 46, D-60528 Frankfurt am Main, Germany

Tel: + 496996769 736; fax: +49 6996769 327;

e-mail: danko.nikolic@gmail.com

Received 19 March 2010 accepted 13 April 2010

marked state dependence [3,12]. As our analysis revealed systematic relations between stimulus direction and oscillation frequency, we also analyzed whether these relations remained constant despite state dependent changes in oscillation frequency.

\section{Materials and methods Anaesthesia and surgical procedures}

We recorded neuronal responses from four cats (three male, one female). Anaesthesia, multisite recordings with Michigan probes, and spike sorting were made with the methods reported in earlier studies [13]. All procedures were conducted in accordance with the guidelines of the Society for Neuroscience and the German laws of animal protection, approved by the ethical committee of the local government and supervised by a veterinarian.

eyes [6], and that the oscillation frequency of local field potentials in adult macaque monkeys was inversely correlated with the size of a moving grating (i.e. a pattern made of multiple bars) [10]. In contrast, intracellular recordings in the visual cortex of kittens suggested no correlation between the oscillation frequency of the membrane potential and spike trains on the one hand, and the contrast, the stimulus direction (only the optimal and orthogonal to optimal directions were investigated), or the position of the moving bar, on the other hand [11]. No data are available on the effect of stimulus direction on oscillation frequencies in adult cats. In this study we address this question and analyze in detail the relations between the stimulus direction and oscillation frequency. The amplitude and frequency of neuronal oscillations exhibit a

\section{Stimulation and recording}

The visual stimuli consisted of high contrast $(C=0.94)$ sinusoidal gratings presented by ActiveSTIM (www.actives tim.com). The gratings moved orthogonal to their orientations in 12 different directions, equally dividing a $360^{\circ}$ circle in $30^{\circ}$ steps $\left(0^{\circ}, 30^{\circ}, 60^{\circ}, 90^{\circ} \ldots 330^{\circ}\right)$. The spatial frequency, size and moving speed of the gratings were $2.4 \%$ cycle, $12^{\circ}$ and $2 \%$, respectively. The receptive fields of the simultaneously recorded units always overlapped, producing clusters spanning up to approximately $10^{\circ}$ of visual angle, over which the stimuli were centered. The gratings were presented for $4 \mathrm{~s}$ with an inter-stimulus interval of $1 \mathrm{~s}$ with their drift directions changing randomly. 
Gratings drifting in the same direction were shown 20 times. Thus, a recording block consisted of 240 stimulus presentations and lasted approximately $20 \mathrm{~min}$. To test the state dependence of oscillation frequencies over a longer period of time, recording blocks were repeated 2-4 times, spanning up to $21.6 \mathrm{~h}$.

In total, 40 single units were isolated. To analyze the oscillatory spiking activity, we calculated auto-correlograms for the time period of $0.3-3.5 \mathrm{~s}$ after the stimulus onset, with the lag ranging from -80 to $+80 \mathrm{~ms}$. The auto-correlograms were then smoothed by a moving average:

$$
\hat{y}_{t}=\frac{y_{t-1}+y_{t}+y_{t+1}}{3}
$$

where $y_{t}$ are the coincidence counts in the bins of the raw auto-correlogram, and $\hat{y}_{t}$ are those of the smoothed auto-correlogram. The smoothed auto-correlograms encompassed thus time lags of $-79 \leq t \leq 79 \mathrm{~ms}$. The estimates of oscillation frequencies were obtained next by fitting the smoothed auto-correlograms with a general Gabor function [14].

Not all auto-correlograms had sufficient counts of coincident events and/or sufficiently strong oscillatory modulation to yield a reliable estimate of the oscillation frequency. The fit of an auto-correlogram was considered sufficiently good to warrant further analysis if $r^{2} \geq 0.8$; and the autocorrelogram was regarded as oscillatory if it exhibited an Oscillation score $\geq 5$ [15].

Out of the 40 single units initially isolated, the autocorrelograms of 25 fulfilled these requirements in at least two stimulation conditions. Out of these, 19 could be tracked continuously over at least two blocks (up to four; average 2.5). Thus, oscillation frequencies of 19 neurons were investigated across 2-4 blocks separated by $40 \mathrm{~min}-$ $21.6 \mathrm{~h}$ and in responses to $2-6$ stimulation conditions (average: 3.1 ).

The standard error of the measured oscillation frequency was estimated by a bootstrap method [16]. For each autocorrelogram, the corresponding bootstrap auto-correlograms were produced by independent random resampling of the 20 original trials. On account of the sampling with replacement, in each bootstrap, the same trial can be drawn multiple times whereas other trials may not be included in a resample. For each auto-correlogram, we generated in total 200 bootstraps and the oscillation frequency was estimated for each. In some cases, the frequency could not be estimated because of the noisiness of the bootstrapped auto-correlograms (usually due to low firing rates). Hence, the resample size was sometimes smaller than 200, ranging between 82 and 200 (average: 178.8). The standard deviation of the bootstrap measurements was used as the estimate of the standard error of the oscillation frequency obtained for the auto-correlogram [16].

We needed to develop a significance test to investigate the effects of stimulus direction and state on the oscillation frequency. We estimated the probability that the highest oscillation frequency would coincide with the highest rate responses. The probability that this will occur by chance equals the reciprocal of the number of available stimulation conditions, $c$. Thus, for a neuron $i$, in a single recording this probability is $p_{i}=1 / c_{i}$. When multiple recordings are made, and if independence between them is assumed, the chance of observing coincidence in all of the recordings is $P_{i}=p_{i}^{r_{i}}$, where $r$ is the number of recordings. Accordingly, the chance that no coincidence will be observed in at least one recording is $1-P_{i}$. Finally, the probability that $n$ out of the total of $m$ neurons $(0 \leq n \leq m)$ exhibit a coincidence in all of the recordings is given by:

$$
P=\sum_{g=1}^{w} \prod_{i=1}^{m} P_{i}^{x_{i j}}\left(1-P_{i}\right)^{1-x_{i j}},
$$

where $w$ is the number of combinations in which $n$ neurons can be taken out of $m$ neurons (i.e. $w={ }^{n} C_{m}$ ), $x_{i g}=1$ if the $i$-th neuron participates in $g$-th combination, and $x_{i g}=0$ otherwise.

We also ranked the stimulation conditions according to the induced frequencies. Consequently, we needed to estimate the chance probability that a given number of neurons would exhibit consistent rankings of stimulation conditions across all recordings. Formula (1) could be used also for this purpose. $P_{i}$ can be computed as $\left(1 / c_{i} !\right)^{r_{i}-1}$, where $c_{i}$ ! is the number of ways $c_{i}$ conditions can be permuted.

\section{Results}

Typically, the oscillation frequencies of neurons varied with the direction of the grating. An example for gratings differing by $30^{\circ}$ in drift direction is shown in Fig. 1 a $(3 \mathrm{~Hz}$ change in the oscillation frequency). The spike waveforms used to sort this unit are shown in Fig. 1b and the post-stimulus time histograms on the basis of which autocorrelograms were computed in Fig. 1c. Overall, the oscillation frequencies with which neurons responded to different stimuli varied by $2.5 \mathrm{~Hz}$ on average, with a maximum of $6.2 \mathrm{~Hz}$. This corresponded respectively to 9.4 and $23 \%$ of the gross average frequency of $26.7 \mathrm{~Hz}$ (averaged across all 48 recording blocks from 19 neurons). The mean standard error of the estimated oscillation frequencies was $0.8 \mathrm{~Hz}$.

Oscillation frequencies tended to be highest when the neurons were presented with the optimal stimulus, that is the stimulus that produced the highest firing rate. For the neuron shown in Fig. 2a, this relation was consistent across three different recording blocks (solid, dashed and dotted lines in Fig. 2a). Altogether, this precise match between oscillation frequency and preferred direction was observed in seven neurons out of 19 (37\%), and consistent across all recording blocks (2-4 recording blocks per neuron); for another two neurons the proportion of recording blocks with a match between oscillation frequency and firing rate was larger than expected by chance (Fig. 2c). 
Fig. 1

(a)
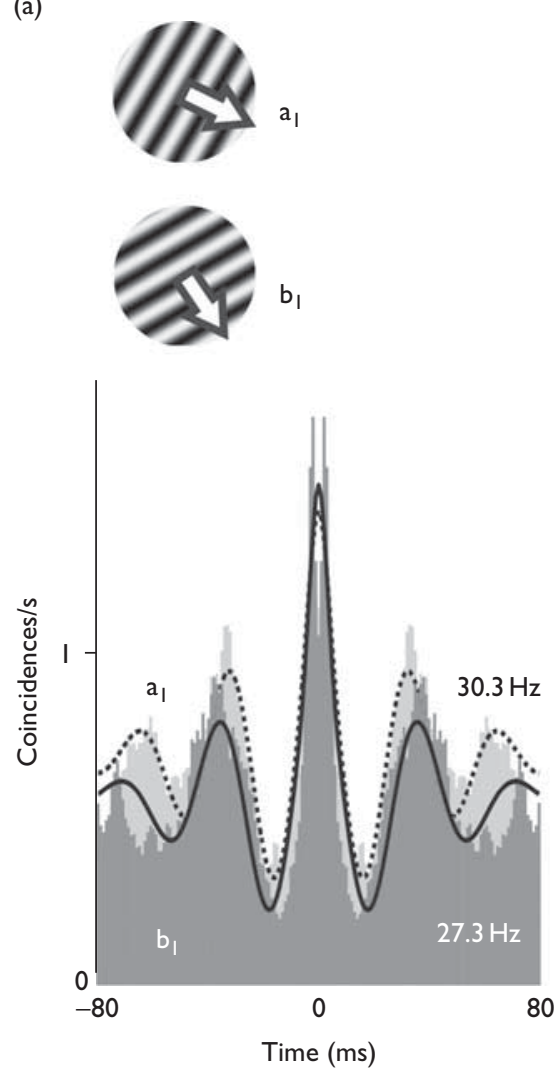

(b)
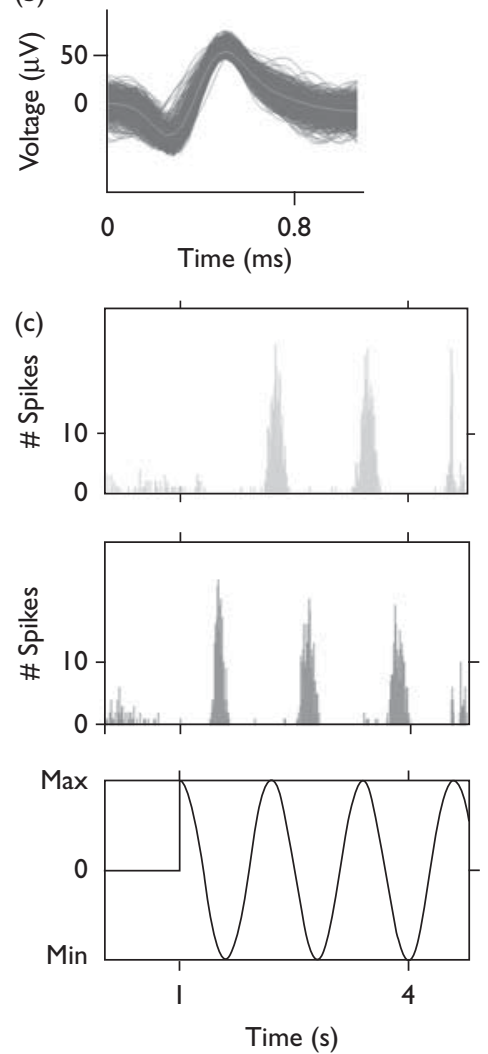

Example change in the oscillation frequency of a neuron as a function of the direction in which a grating stimulus drifts. (a) Auto-correlograms of spike trains obtained in response to two different stimuli $\left(a_{1}\right.$ and $\left.b_{1}\right)$. The method for estimating the oscillation frequency is illustrated by the fitted Gabor functions (dashed and solid curves). The resulting estimates of oscillation frequencies are indicated on the right. (b) The waveforms of the extracellularly recorded action potentials (dark gray envelope) and the average (light grey curve) used for the unit in (a). (c) Peri-stimulus time histograms (PSTH) in response to grating $a_{1}$ (top) and grating $b_{1}$ (middle). Bottom: The stimulus-induced rhythm with which screen pixels change the luminance ( $y$-axis) because of the drift of the gratings along a trial ( $x$-axis).

The probability of obtaining by chance at least seven neurons with exact match between maximal firing rate and frequency is 0.003 (see Materials and methods section for the calculation procedure).

Oscillation frequency did not stay constant across recording blocks. When responses to the same stimuli were recorded several hours apart, the frequency of the oscillatory patterning in the neuron's spiking activity changed by up to $3.2 \mathrm{~Hz}$. For example, in Fig. 2a, the oscillation frequency changed by $1.9 \mathrm{~Hz}$ between the first and the third session. These changes were usually consistent across the entire spectrum of stimuli (see e.g. Fig. 2a). For the example in Fig. 2b oscillation frequency was reduced by approximately $3 \mathrm{~Hz}$ in recording block 4 in comparison with blocks 1-3 across all four stimulus directions.

Despite the changes of the mean oscillation frequencies, the relative modulation as a function of the grating's drift direction remained constant. This relative constancy was independent of the overall changes in the oscillation fre- quency. Hence, in a recording block in which oscillations were predominantly slow, the differences across stimulus conditions were about the same as in a recording session in which the oscillations were overall fast. Examples of 'frequency patterns' representing the changes in the oscillation frequencies for different stimuli are shown for one neuron across three recording blocks in Fig. 2a and for two neurons across four blocks in Fig. 2 b.

To quantify the degree to which the frequency patterns stayed stable over time, we first superimposed them by subtracting the overall mean of the pattern from the oscillation frequency obtained in each stimulus condition (Fig. 2a, inset). Next, we compared the variability of the resulting oscillation frequencies across the stimulus conditions to the variability of responses to the same stimulus condition recorded in different blocks (Fig. 3). In the former case, the standard deviation was on average 3.73 times higher than in the latter. This ratio reduced to 1.89 for condition-shuffled data (crosses in Fig. 3; $P=0.008$, Wilcoxon signed-rank test). This result 
Fig. 2
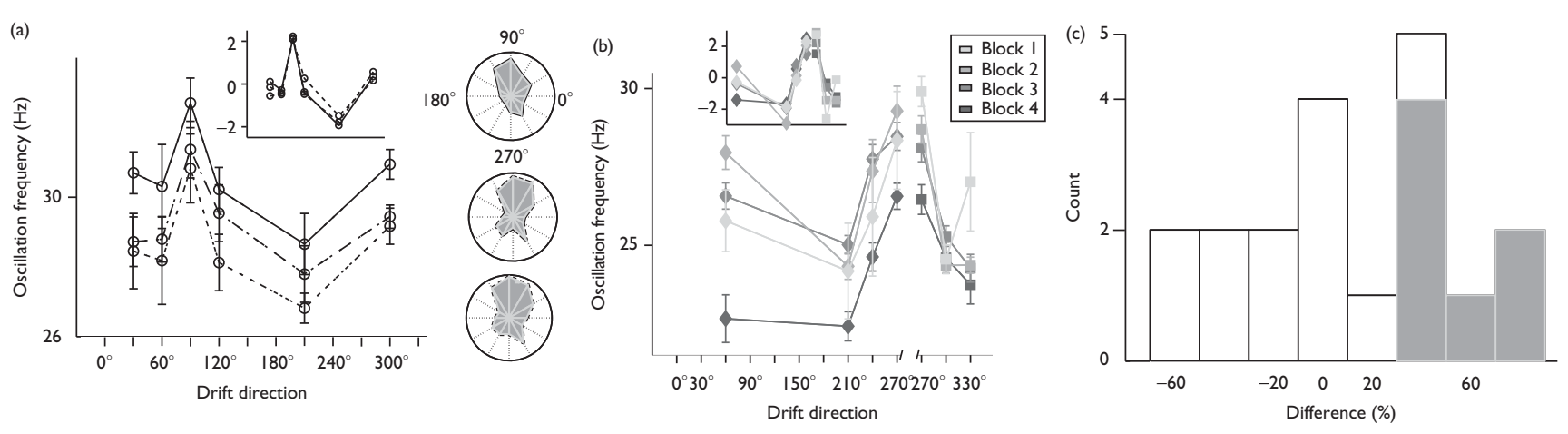

Example changes in oscillation frequencies for three neurons as a function of grating orientation and recording blocks. (a) Oscillation frequencies of one neuron obtained in six stimulation conditions, and in three recording blocks with a total time span of approximately $3 \mathrm{~h}$. Inset: data superimposed by centring the means of the $y$-axis. Axis labels are the same as in the main figure. Right: orientation tuning determined by the firing rates with which the neuron responded to the same set of stimuli, shown separately for all three blocks. (b) Another example of two neurons (diamonds and squares) recorded in the same session, with the total time span across the four recording blocks of $21.6 \mathrm{~h}$. Error bars: standard error of the estimate. Inset: the same as in (a). (c) Chance level vs. observed number of recordings in which maximum rate coincides with maximum oscillation frequency, shown for all 19 neurons. $X$-axis: the difference between the observed percent agreements and that expected by chance. Grey: the seven neurons for which the observed percent agreement was 100\%.

Fig. 3

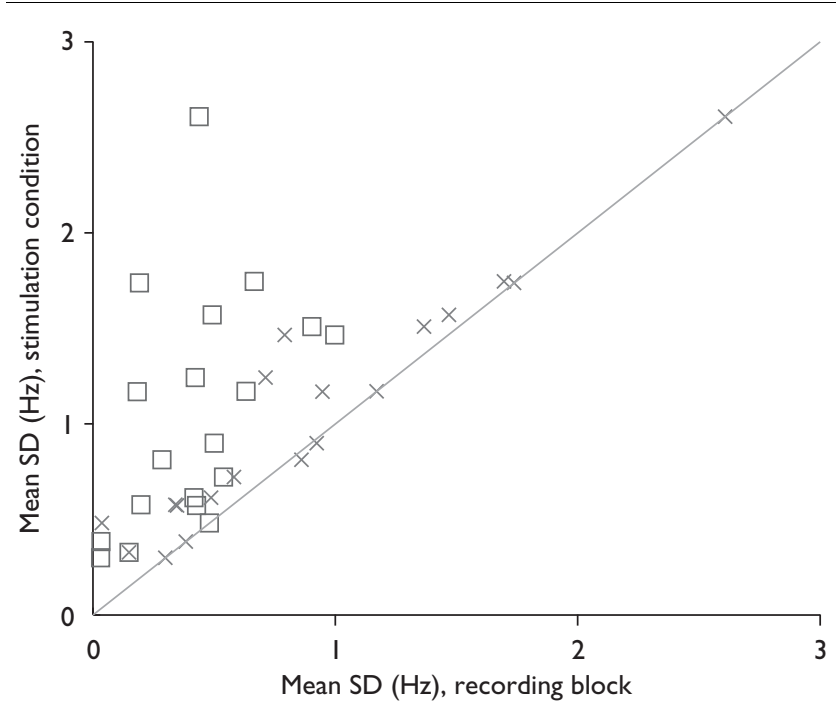

The mean variability of oscillation frequencies across different recording blocks compared to the variability across stimulus conditions, expressed as standard deviation (SD). Squares: original data. Crosses: condition-shuffled control data. Diagonal: identity line.

indicates that, over different recording blocks, the relative relationships within the patterns stayed constant despite the shifts of the mean oscillation frequencies.

Finally, the stability of the patterns was tested also by ranking stimulus directions according to the induced oscillation frequency, and comparing the ranks across recording blocks. For 10 out of 19 neurons (53\%), the ranks of stimulus directions stayed identical. The probability of obtaining this result by chance is less than 0.001 (see Materials and methods section). For two neurons, more than $2 / 3$ of the tracked stimulus directions stayed in the same relative rank across all blocks. For one, all stimulus directions stayed in the same relative rank across more than $3 / 4$ of the tracked blocks. These levels of temporal stability were unlikely to be obtained by chance (in all cases, $P<0.05$; computed from all possible combinations of ranks). Thus, in total, the ranks of 13 neurons $(72 \%)$ could be considered stable across the recording blocks.

\section{Discussion}

In this study we investigated the modulation of oscillation frequency both as a function of stimulus properties and states. To achieve this goal, we had to overcome two difficulties: all investigated neurons had to be clearly identifiable by spike sorting and the neurons had to maintain sufficiently high spike counts across all recordings. We succeeded in collecting a sample of 19 neurons (i.e. single units), which was sufficiently large to obtain statistically significant results.

We found that in many neurons the frequency of induced oscillations increased with increasing discharge rate of light evoked responses. Hence, in some neurons, oscillation frequency reflects, in a similar way as response amplitude, the match between the cells' preferences for stimulus orientation and the direction of motion of the presented stimulus. At present, it is unclear whether this tuning of oscillation frequency is a result of the individual properties of a neuron, example of the resonant properties of its membrane $[17,18]$ and the modulation of resonance by neuromodulators [19], or whether it is a network effect [20,21]. The coherence of the input may play a role too [22]. In any case, our data indicate that oscillation frequencies of individual neurons or groups of neurons with similar response preference must be 
adjustable selectively. Considering that a drifting grating activates large volumes of cortical tissue containing both optimally and sub-optimally stimulated neurons, this predicts a dispersion of phases, the optimally stimulated population oscillating at higher frequencies than the less activated populations. As recordings suggest that drifting gratings induce global oscillations that are synchronized on average with zero phase delay across visual cortex $[23,24]$, one expects the discharges of optimally activated cells to exhibit changes in the firing times relative to the global oscillatory rhythm or at least relative for less optimally stimulated cells. This cell-specific adjustment of oscillation frequency on top of a global oscillatory patterning of activity resembles closely, and may be related to the recently discovered mechanism underlying the phenomenon of phase precession in the hippocampus [25]. Whether these time delays are used for the encoding of visual information, as is likely the case for the encoding of spatial information in the hippocampus, is presently unknown. The present results suggest that future investigations should take into account also the oscillations frequencies of individual neurons. Interestingly, the adjustments in the frequency of oscillations may be only a property of mature brains because stimulusinduced changes could be found only in adult cats, as used in our experiment, but not in kittens, as reported elsewhere [11].

The finding that the overall oscillation frequency can change over time, as a state variable, and yet the tuning remains stable, has implications for models of beta/ gamma oscillations [12]. Future models should allow for both types of changes - the state-dependent and the stimulus-dependent ones. Hence, our findings emphasize the point that the dynamical changes in the frequencies of oscillatory rhythms are important for the understanding of brain dynamics - a variable that has apparently not received the due attention until now.

\section{Conclusion}

The oscillation frequency with which neurons respond to drifting sinusoidal gratings is not constant but changes as a function of stimulus direction. More optimal stimuli have a tendency to induce higher oscillation frequencies, that is, the firing rate tuning of neurons is mirrored by the tuning of their oscillation frequencies. Moreover, under anesthesia, the average oscillation frequency changes over time. These changes in the baseline frequency do not affect the tuning properties of the responses.

\section{Acknowledgements}

This study is supported by a DFG grant number NI 708/ 2-1. In addition, support came from the Hertie Foundation, and the German Federal Ministry of Education and Research (BMBF) within the 'Bernstein Focus: Neurotechnology' through research grant 01GQ0840.

\section{References}

1 Gray CM, Singer W. Stimulus-specific neuronal oscillations in orientation columns of cat visual cortex. Proc Natl Acad Sci U S A 1989; 86: 1698-1702.

2 Jagadeesh B, Gray C, Ferster D. Visually evoked oscillations of membrane potential in cells of cat visual cortex. Science 1992; 257:552-554.

3 Herculano-Houzel S, Munk M, Neuenschwander S, Singer W. Precisely synchronized oscillatory firing patterns require electroencephalographic activation. J Neurosci 1999; 19:3992-4010.

4 Chabli A, Guitton D, Fortin S, Molotchnikoff S. Cross-correlated and oscillatory visual responses of superficial-layer and tecto-reticular neurons in cat superior colliculus. Exp Brain Res 2000; 131:44-56.

5 Traub D, Bibbig A, LeBeau F, Buhl E, Whittington M. Cellular mechanisms of neuronal population oscillations in the hippocampus in vitro. Annu Rev Neurosci 2004; 27:247-278.

6 Gray C, Viana Di Prisco G. Stimulus-dependent neuronal oscillations and local synchronization in striate cortex of the alert cat. J Neurosci 1997; 17:3239-3253.

7 Sannita WG. Stimulus-specific oscillatory responses of the brain: a time/frequency-related coding process. Clin Neurophysio/ 2000; 111:565-583.

8 Buzsáki G, Draguhn A. Neuronal oscillations in cortical networks. Science 2004; 304:1926-1929.

9 Tallon-Baudry $\mathrm{C}$. The roles of gamma-band oscillatory synchrony in human visual cognition. Front Biosci 2009; 14:321-332.

10 Gieselmann M, Thiele A. Comparison of spatial integration and surround suppression characteristics in spiking activity and the local field potential in macaque V1. Eur J Neurosci 2008; 28:447-459.

11 Bringuier V, Frégnac Y, Baranyi A, Debanne D, Shulz D. Synaptic origin and stimulus dependency of neuronal oscillatory activity in the primary visual cortex of the cat. J Physiol 1997; 500:751-774.

12 Nikolic D. Model this! Seven empirical phenomena missing in the models of cortical oscillatory dynamics. Proceedings of the International Joint Conference on Neural Networks (in press) 2009.

13 Biederlack J, Castelo-Branco M, Neuenschwander S, Wheeler DW, Singer W, Nikolić D. Brightness induction: rate enhancement and neuronal synchronization as complementary codes. Neuron 2006; 52:1073-1083.

14 König P. A method for the quantification of synchrony and oscillatory properties of neuronal activity. J Neurosci Methods 1994; 54:31-37.

15 Muresan RC, Jurjup O, Moca V, Singer W, Nikolić D. The oscillation score: an efficient method for estimating oscillation strength in neuronal activity. J Neurophysiol 2008; 99:1333-1353.

16 Efron B, Tibshirani R. Bootstrap methods for standard errors, confidence intervals, and other measures of statistical accuracy. Statist Sci 1986; 1:54-75.

17 Maex R, De Schutter E. Resonant synchronization in heterogeneous networks of inhibitory neurons. J Neurosci 2003; 23:10503-10514.

18 Tohidi V, Nadim F. Membrane resonance in bursting pacemaker neurons of an oscillatory network is correlated with network frequency. $J$ Neurosci 2009; 29:6427-6435.

19 Ito $\mathrm{H}$, Schuman E. Frequency-dependent signal transmission and modulation by neuromodulators. Front Neurosci 2008; 2:138-144.

20 Brunel N, Wang $X$. What determines the frequency of fast network oscillations with irregular neural discharges? I. Synaptic dynamics and excitation-inhibition balance. J Neurophysio/ 2003; 90:415-430.

21 Whittington MA, Traub RD, Kopell N, Ermentrout B, Buhl EH. Inhibition-based rhythms: experimental and mathematical observations on network dynamics. Int J Psychophysiol 2000; 38:315-336.

22 Burwick T. Temporal coding: assembly formation through constructive interference. Neural Comput 2008; 20:1796-1820.

23 Nikolić D. Non-parametric detection of temporal order across pairwise measurements of time delays. J Comput Neurosci 2007; 22:5-19.

24 Schneider G, Havenith M, Nikolić D. Spatio-temporal structure in large neuronal networks detected from cross-correlation. Neural Comput 2006; 18:2387-2413.

25 Harvey C, Collman F, Dombeck D, Tank D. Intracellular dynamics of hippocampal place cells during virtual navigation. Nature 2009; 461:941-946. 